\title{
Rapid Identification of Stress-Related Fingerprint from Whole Bacterial Cells of Bifidobacterium lactis Using Matrix Assisted Laser Desorption/Ionization Mass Spectrometry
}

\author{
Laure F. Marvin-Guy, Stephan Parche, Sandrine Wagnière, Julie Moulin, \\ Ralf Zink, Martin Kussmann, and Laurent B. Fay \\ Nestlé Research Center, Nestec Ltd., Lausanne, Switzerland
}

\begin{abstract}
Whole cells of Bifidobacterium lactis were analyzed by matrix-assisted laser desorption/ ionization time-of-flight mass spectrometry (MALDI-TOFMS). Characteristic and reproducible mass spectra were obtained in the mass range from 6 to $19 \mathrm{kDa}$. After several days of bacterial cell storage at $4{ }^{\circ} \mathrm{C}$ (D0, D2, and D6), only minor signal differences were observed. Under identical and reproducible conditions, fourteen relevant diagnostic ions were identified. Moreover, control- and stress-related fingerprints were rapidly obtained using MALDITOFMS by comparison of protein patterns obtained from non-stressed (control) versus stressed cells (addition of bile salts during growth). After quantitative validation of the MALDI-MS data by a statistical approach, two and eight signals were assigned as control- and stress-specific ions, respectively. This work provides the evidence that MALDI-TOFMS can be used for the identification of stress-related fingerprint of B. lactis bacterial cells and could have a high potential for the assessment of the physiological status of the cells. (J Am Soc Mass Spectrom 2004, 15, 1222-1227) @ 2004 American Society for Mass Spectrometry
\end{abstract}

$\mathrm{M}$ atrix-assisted laser desorption/ionization mass spectrometry (MALDI-TOFMS) has become a popular and versatile method of analyzing a broad range of macromolecules (proteins, DNA, oligonucleotides, oligosaccharides) from biological origin [1]. MALDI-TOFMS has been used during the past years for the direct determination of proteins in a complex biological mixture without prior separation at high sensitivity levels (i.e., low femtomoles). In particular, MALDI mass spectrometry has been described for generating marker profiles directly from unfractionated micro-organisms such as viruses, bacteria, fungus cells, and spores [2-11]. This approach enables detection, identification and characterization of peptides and proteins from "intact" micro-organisms and was applied in various fields such as biotechnology, cell biology, and pharmaceutical research [7]. For instance, specific and unique mass spectral fingerprint allowed the identification of "intact" gram-negative and gram-positive bacteria taken from culture [11].

Other studies have focused on the assignment of

Published online July 2, 2004

Address reprint requests to Dr. L. F. Marvin-Guy, Nestlé Research Center, Nestec Ltd., Vers-chez-les-Blanc, 1000 Lausanne 26, Switzerland. E-mail: laure.marvin-guy@rdls.nestle.com markers on family, genus, species, and even on strain levels. As an example, F. Leenders et al. used MALDITOFMS to obtain rapid and efficient typing of six Bacillus subtilis strains [12].

However, reproducible sample preparation and mass spectra acquisition are difficult to achieve and often the reproducibility of mass spectral results derived from experiments involving MALDI protein/ peptide ionization directly from cells is poor. Thus, the sample preparation strategy adopted for MALDITOFMS of whole bacteria is crucial to improve this reproducibility. Indeed, several studies have shown that the obtained bacterial profiles depend on a number of parameters, such as instrumentation, culture media, and bacterial growth [13-18].

The present work focuses on Bifidobacterium lactis NCC 362. Bifidobacteria are important components of the human intestinal microflora, occurring at concentration levels of $10^{9}$ to $10^{10}$ cells/g of feces [19]. They are also included in various infant formulae and fermented dairy products, added mainly for their health-promoting activity. Despite their importance for the food industry, the knowledge of bifidobacterial molecular mechanisms, e.g., probiotic functions or bile resistance, is still limited.

The aim of this study was (1) to evaluate the MALDI 


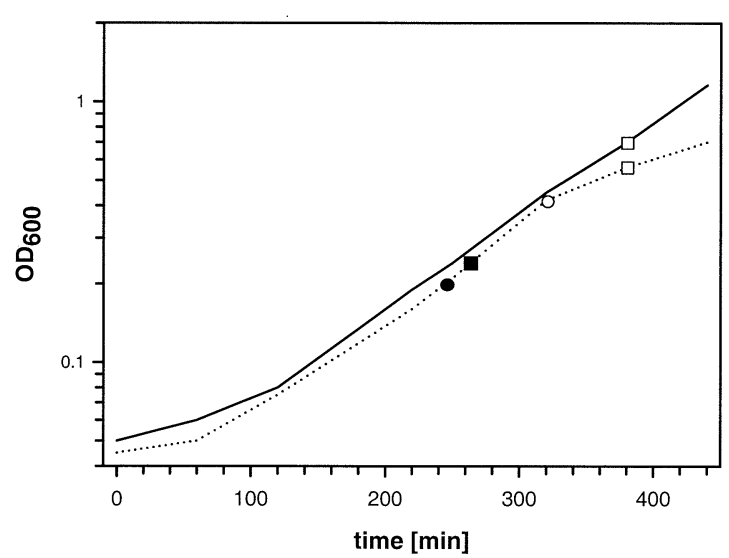

Figure 1. Typical growth curves of B. lactis in MRS + Cys of control (without bile, solid line) and stress (presence of bile, dashed line). Filled circle: a final concentration of $0.1 \%$ bile was added; filled square: $100 \mathrm{ml}$ of the culture were harvested (adapted cells); open circle: a final concentration of $1 \%$ bile was added; open square: $100 \mathrm{ml}$ of the cultures were harvested (stressed and control cells).

mass spectral reproducibility, using the same cell preparation even after storage for several days at $4{ }^{\circ} \mathrm{C}$, (2) the comparison of MALDI-TOF mass spectral profiles from whole bacteria under defined physical conditions (non-stressed and bile-salt stressed) in order to monitor changes in the protein profile, and (3) to identify relevant control- and stress-related fingerprints. All MALDI-MS data obtained were quantitatively validated by a statistical approach.

\section{Experimental}

\section{Bacterial Growth and Sample Preparation}

Cells of Bifidobacterium lactis NCC 362 (Nestlé Culture Collection) were grown under anaerobic conditions at $37{ }^{\circ} \mathrm{C}$ using de Man Rogosa Sharpe medium (MRS, Difco, Chemie Braunschwig, Basel, Switzerland) supplemented with $4 \mathrm{mM}$ L-cysteine-HCl (MRS+Cys). Two culture media (control and stress) were incubated in a glovebox system (Coy Laboratories, Grass Lake, MI) in an atmosphere of $5 \% \mathrm{CO}_{2}, 10 \% \mathrm{H}_{2}$, and $85 \% \mathrm{~N}_{2}$ under continuous stirring (200 rpm). Cell growth was monitored by measuring the optical density at a wavelength of $600 \mathrm{~nm}\left(\mathrm{OD}_{600}\right)$. Bile salts (oxgall, Difco) were added to a growing stress culture at final proportions of 0.1 and $1.0 \%$ at $\mathrm{OD}_{600}$ of 0.2 and 0.4 , respectively, while the control culture remained untreated (Figure 1). After incubation $\left(10^{\prime}\right.$ for $0.1 \%$ and $60^{\prime}$ for $1 \%$ of bile), cultures were chilled on ice and $100 \mathrm{ml}$ of each culture harvested were centrifuged $\left(5000 \mathrm{rpm}, 4{ }^{\circ} \mathrm{C}, 10 \mathrm{~min}\right)$, washed twice with ice-cold deionized water and were adjusted to the same OD. Cell pellets were stored at $-20{ }^{\circ} \mathrm{C}$. For MALDI mass spectra reproducibility experiments, bacterial samples were stored at $4{ }^{\circ} \mathrm{C}$ in deionized water and MALDI analysis were performed after Day 0 (D0), Day 2, (D2), and Day 6 (D6) to asses the eventual protein degradation.

\section{MALDI-TOFMS}

Mass spectra were recorded on an Autoflex (Bruker, Bremen, Germany) MALDI time-of-flight mass spectrometer operating in delayed extraction linear positive ion mode. Sinapinic acid was used as matrix with a saturated solution of acetonitrile $(30 \%)$ and $0.1 \%$ TFA in water $(70 \%)$. Frozen cell pellets (about $100 \mathrm{mg}$ ) were thawed on ice and resuspended in $400 \mu \mathrm{L}$ cold deionized water. One $\mu \mathrm{l}$ (about $0.20 \mathrm{mg}$ ) of sample was diluted with $19 \mu \mathrm{L}$ of $0.1 \%$ TFA aqueous buffer. Typically, $1 \mu \mathrm{L}$ of the saturated matrix solution was mixed with $1 \mu \mathrm{L}$ of the diluted bacterial sample (from $5 \times 10^{5}$ to $10^{6}$ cells). The resulting mixture was then deposited on a "ground steel" (Bruker) target and allowed to dry at room temperature. Ions formed upon irradiation by a pulsed UV laser beam (nitrogen laser, $337 \mathrm{~nm}$ ) were accelerated at $20 \mathrm{kV}$. Each mass spectrum was produced by averaging from 70 to 100 laser shots spread all over the spot surface. External calibration was performed with a protein mixture containing bovine insulin, ubiquitin, cytochrome $c$, and myoglobin (Bruker). The mass accuracy obtained by external calibration in linear mode was approximately $0.05 \%$. It is worthwhile to mention that MALDI mass spectra obtained with independent culture gave the same tendency and highly similar mass fingerprints.

\section{Statistical Analyses}

For relative quantitation, absolute ion intensities cannot be compared between MALDI-MS experiments. Therefore, MS data need to be rescaled in order to be comparable. Thus, the most abundant ion from the control sample $(8113 \mathrm{kDa})$ at D0 was defined as the reference peak. All absolute intensities of a sample were divided against the absolute intensity of the reference peak. All statistical analyses were performed on rescaled intensities. For all comparisons, differences were assessed by the t-test. Depending on the data distribution, either the equal variance t-test or the Aspin-Welch unequal variance t-test was used. The software used was NCSS 2001 by Jerry Hintze, Number Cruncher Statistical Systems, Kaysville, UT.

\section{Results and Discussion}

\section{Reproducibility Tests}

Identifying bacteria by their mass spectral fingerprints is of practical significance only if the mass spectra of the same sample and identical culture media are reproducible. To further optimize the analysis reproducibility of whole Bifidobacterium lactis cells by MALDI-TOFMS, different sample preparations were tested, such as various washing steps of bacterial cells, cell dilution in $0.1 \%$ TFA, and usage of different MALDI matrices. The most reproducible mass spectra were obtained using the conditions outlined in the experimental part. Figure 

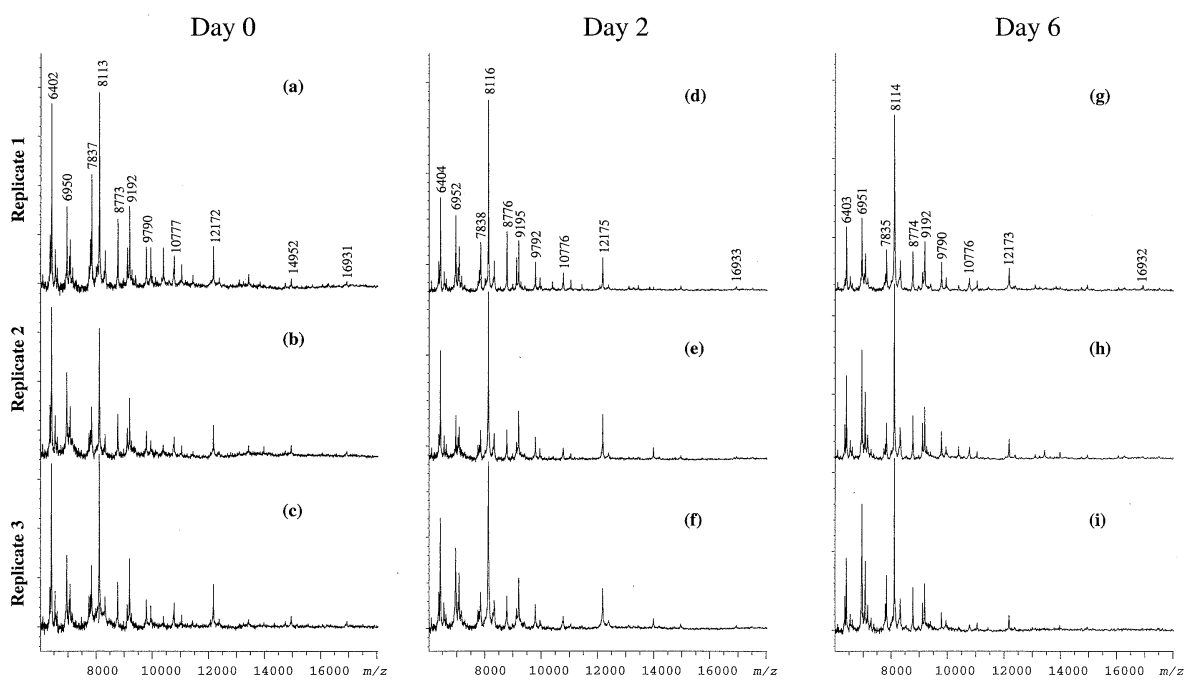

Figure 2. Replicate MALDI mass spectra of $B$. lactis control sample after 0,2 , and 6 days of storage at $4{ }^{\circ} \mathrm{C}$. Triplicate analyses are shown in (a), (b), and (c) windows. Sinapinic acid was used as matrix.

2 shows the MALDI mass spectra of $B$. lactis obtained before and after sample storage for several days at $4{ }^{\circ} \mathrm{C}$. The reproducibility of mass spectral fingerprints from whole bacteria was demonstrated by three independent mass spectra for each day.

At D0, the five most dominant ions are observed at $\mathrm{m} / \mathrm{z}$ 6402, 6950, 7837, 8113, and 9192 (Figure 2a-c). At D2, the most dominant signals are observed at $\mathrm{m} / \mathrm{z}$ 6404, 6952, 8116, 8776, and 9195 (Figure 2d-f), whereas at D6 they were $\mathrm{m} / \mathrm{z}$ 6403, 6951, 8114, 8774, and 9192 (Figure $2 \mathrm{~g}-\mathrm{i}$ ).

Nevertheless, for each day mass spectra acquired are highly similar to each other demonstrating the reproducibility per day. In order to strengthen and quantitatively validate this result, a statistical approach was applied to three replicates obtained at D0. Among the total ions observed, we have decided to consider only relevant signals above a certain cut-off, i.e., peaks with a signal-to-noise ratio greater than 15 . Fourteen ions were selected at $m / z 6347,6403,6535,6951,7073,7836$, 8115, 8321, 8774, 9114, 9193, 9791, 10776, and 12173 $( \pm 0.05 \%)$. These signals are considered as relevant diagnostic ions for Bifidobacterium lactis NCC 362.

Figure 3 presents a graph with one replicate as function of the mean. In this graph, the x-axis represents the mean values of absolute intensities for the fourteen most dominant ions, the $y$-axis the value of the replicate for each peak. If the measurement was perfectly reproducible, the data points of all the graphs should be aligned on the " $x=y$ " line, which would represent the equality between the mean and the replicate. The overall reproducibility is demonstrated in Figure 3. Indeed, the replicate data points are very close to the " $x=y$ " line and it is the same for the other replicates. Furthermore, a very good correlation coefficient $\left(R^{2}\right)$ above 0.98 $(n=3)$ was obtained among the three replicates performed on the control sample at D0.

When comparing the replicates of the three different days (D0, D2, and D6), the MALDI mass spectra revealed highly similar mass signals. However, it should be further noted that besides the high similarity of the mass spectral fingerprints, differences in signal intensity occurred at $m / z$ 6403, 9193, and 12173 that exhibit lower intensities at D2 and D6 compared with the ones at D0. These differences might be due to the variability of the dried sample spot and/or to the partial protein degradation. These results are in agreement with observations of previous studies [14, 16, 20].

Using the statistical approach, the signal intensity differences between D0 and D2 (D0-D2) as well as D0 and D6 (D0-D6) are reported in Figure 4 for thirteen ions (8113 is the reference for rescaling). From this graph, it appeared that for most of the ions no big changes were detected between the different days. Indeed, only three ions at $\mathrm{m} / \mathrm{z}$ 6403, 9193, and 12173 showed statistically significant differences. For the former two ions, the intensities are statistically lower at D2 and D6 compared with D0, whereas for the latter, the signal decrease became significant at D6.

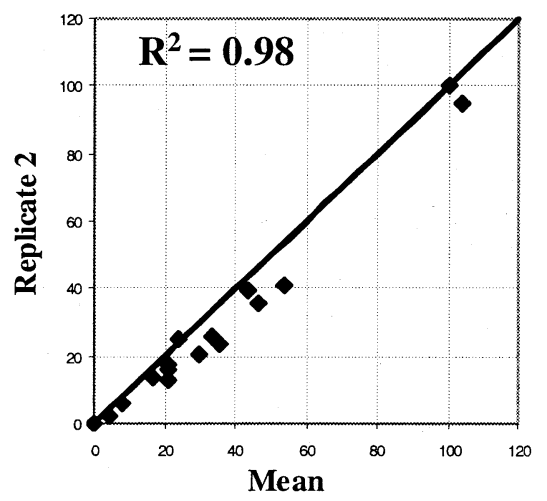

Figure 3. On replicate as function of the mean on control cells of B. lactis at day 0. x-axis: mean values of absolute intensities for each ion; $y$-axis: value of one replicate. 


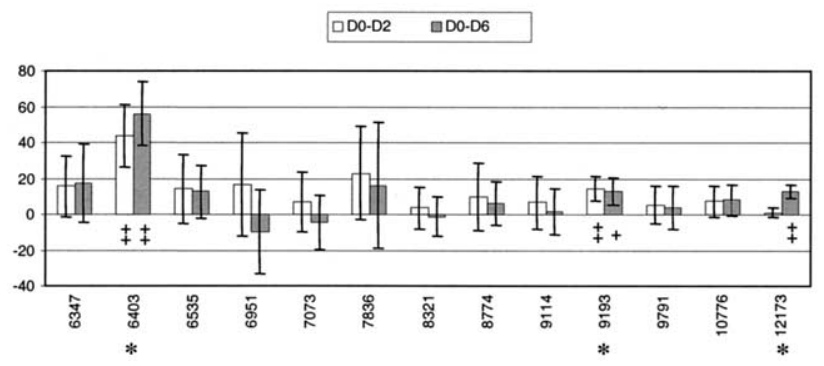

Figure 4. Typical Rescaled ion intensity differences between: (white bar) D0 and D2 ; (filled bar) D0 and D6, with confidence intervals at $95 \%$. Asterisk denotes masses which correspond to the peaks showing statistic differences between D0, D2, and D6. Statistically significant $+: p<0.05 ;++: p<0.01 ;+++: p<0.001$.

Despite these minor signal differences, the reproducibility of the MALDI spectra for each day or during a period of time was demonstrated for B. lactis bacteria. Nevertheless, in order to compare different samples under the best identical and reproducible conditions, we observed the utility of analyzing them the same day and under the same conditions in order to reduce slight fluctuation linked to dried sample spot variability and/or protein degradation.

\section{Stress-Related Fingerprint Identification}

In our study, we were interested in studying the possibility of identifying stress-related fingerprint from whole cells grown in the presence of different bile salt concentrations. Figure 5 depicts one of the replicate MALDI mass spectra obtained from whole cells grown under three different conditions (see also Figure 1), control (without bile, Figure $5 \mathrm{a}$ ), preadaptation $(0.1 \%$ bile, Figure $5 b$, and stress ( $1 \%$ bile, Figure $5 c)$. For the control sample, we noticed again that the most dominant ions were observed at $\mathrm{m} / \mathrm{z} 6402,6950,7837,8113$, and 9192, and at $\mathrm{m} / \mathrm{z} 6347,6402,6535,6950,7072$, and 8115 for the preadaptation condition. For the bilestressed bacteria (Figures 5c), the most dominant signals were at $m / z$ 6403, 6950, 7072, 8114 and 8773.

In the lower mass range of the three spectra (from 6 to $11.5 \mathrm{kDa}$ ) some differences could be observed between control, preadapted and stressed cells but it was very difficult to quantify them because no real trend was observed. When the MALDI spectra were expanded in the range of 11.5 to $16.5 \mathrm{kDa}$, prominent ions were observed at $\mathrm{m} / \mathrm{z} 12172,13978$, and 14953 for the control and at $\mathrm{m} / \mathrm{z} 12172,13978$ for the preadaptation condition. Interestingly, the peak at $\mathrm{m} / \mathrm{z} 14953$ is missing in the mass spectrum obtained from cells containing $0.1 \%$ bile. In the same expanded mass range, stressed cells ( $1 \%$ bile) clearly exhibited four new additional ions observed at $\mathrm{m} / \mathrm{z}$ 12064, 13095, 16060, and 16278 (Figure 5c) compared to the control and preadaptation conditions. These four new signals were clearly observed in several replicate experiments and were therefore designated as stress-specific ions for B. lactis.

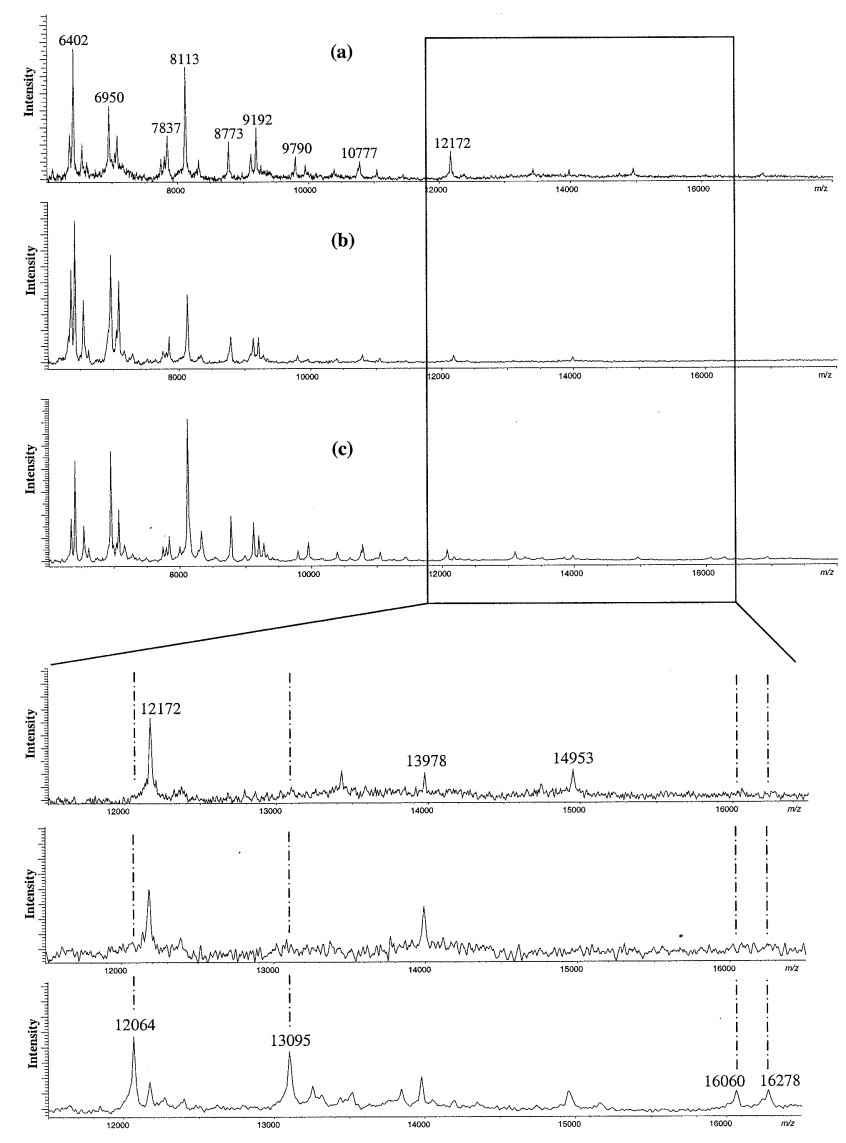

Figure 5. Typical MALDI mass spectra of B. lactis whole cells: (a) control, (b) $0.1 \%$ bile salts, (c) $1 \%$ bile salts. Sinapinic acid was used as matrix. Three independent triplicate mass spectra were performed for each condition.

These results have also been validated and quantified by statistical treatments for the two distinct mass ranges (i.e., $6-11.5$ and $11.5-16.5 \mathrm{kDa}$ ). To better compare our MS data, the ion intensities, after being rescaled against the most intense one (i.e., $8113 \mathrm{Da}$ ), were plotted to show differences between control and stressed cells (Figure 6). In the lower mass range (i.e., $6-11.5 \mathrm{kDa})$, no significant difference was observed for $m / z$ 6402, 7837, 8320, 8773, 9114, 9790, and 10777. However, five other ions out of 13 showed significant statistical differences in signal intensity between control and stress samples (Figure 6a). Indeed, the ion intensities of $m / z 6347,6534,6950$, and 7072 are higher in the stressed cells compared with the control cells whereas for $m / z$ 9192, the intensity is lower in the stressed cells compared to the control sample. Consequently, these five signals could be considered as stress-specific ions and $m / z 9192$ as control-specific ion.

For the expanded higher mass range (i.e., 11.5-15.5 $\mathrm{kDa}$ ), among the seven ions observed, peaks at $\mathrm{m} / \mathrm{z}$ 12064, 13095, 16060, and 16278 have significantly higher intensities in the stressed cells compared to the control cells (Figure 6b). Conversely, the ion intensity at $\mathrm{m} / \mathrm{z}$ 12172 is higher in the control sample. Thus, in the 


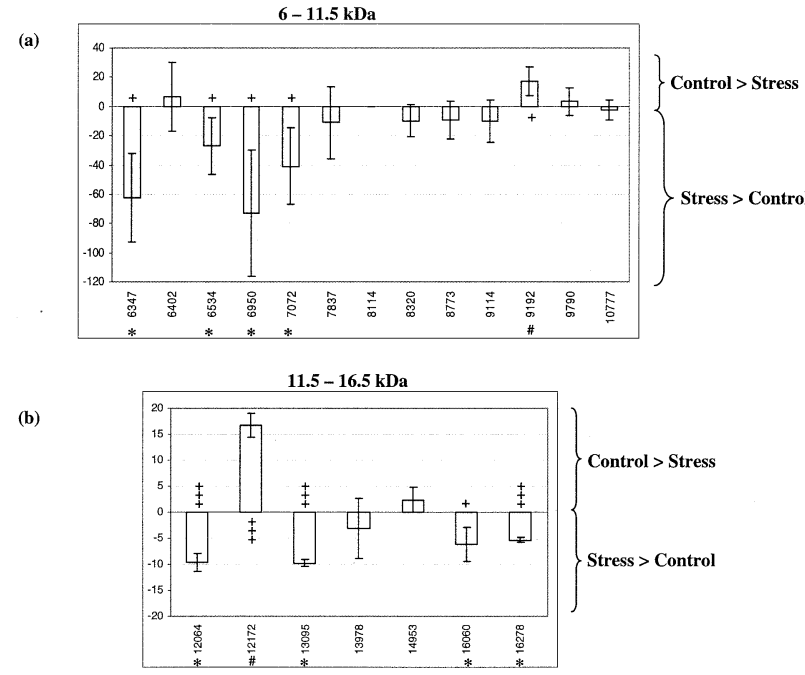

Figure 6. Typical Rescaled ion intensity differences between control and stressed cells from (a) 6 to $11.5 \mathrm{kDa}$ and (b) 11.5 to 16.5 $\mathrm{kDa}$, with confidence intervals at $95 \%$. The pound symbol and the asterisk highlight the masses showing statistic differences and corresponding to peak intensities of Control $>$ Stress and Stress $>$ Control, respectively. Statistically significant $+: p<0.05 ;++: p<$ $0.01 ;+++: p<0.001$.

higher mass range, four signals in the stressed sample spectrum could be considered as relevant stress-specific ions whereas $\mathrm{m} / \mathrm{z} 12172$ could be monitored for controlspecific ion.

Moreover, it should be noted that variations in ion intensities are much more significant in the lower than in the higher mass range. Indeed, the coefficient of variation observed for several replicate experiments of the different ions apart $m / z 16060$ ranged from 4 to $11 \%$ and were much lower compared to those observed in the lower mass range (Figure 6a). Indeed, in this range (6-11.5 kDa), the five signals $(m / z$ 6347, 6535, 6950, 7072, and 9192) identified as control and stress-specific ions by statistical approach, showing statistically significant differences between control and stressed cells, gave coefficient of variation of $31,44,37,40$, and $35 \%$, respectively. For this reason, the visible evidence in the MALDI mass spectra rendered the stress-related fingerprint identification in this low mass region very difficult.

\section{Conclusions}

Our work describes the first identification of stressrelated fingerprint in bifidobacteria by MALDI-TOFMS. The reproducibility of the MALDI-MS measurements has been demonstrated and validated by a statistical approach. Under identically reproduced experimental conditions, i.e., treatment of cells and sample preparation for MS, fourteen signals were identified as relevant diagnostic ions for B. lactis NCC 362.

Moreover, we compared whole cells of B. lactis, which were grown in the absence and presence of bile salts and subjected them to direct MALDI-TOFMS analysis. After quantitative validation of the MALDI-MS data by a statistical approach, two and eight signals were assigned as control- and stressspecific ions, respectively. The five peaks $(\mathrm{m} / \mathrm{z} 12064$, $12172,13095,16060$, and 16278) identified as relevant stress-specific ions in the higher mass range (11.5-16.5 $\mathrm{kDa}$ ) were observed with a low coefficient of variation, which confirmed the visible evidence in the MALDI mass spectra.

This result provides the evidence that MALDITOFMS can be used for the identification of bile stressrelated fingerprint of $B$. lactis whole bacterial cells and could have a high potential for the assessment of the physiological status of the cells.

\section{Acknowledgments}

The authors thank A. Mercenier for critical reading; they also thank C. Parker and B. Viret for proof-reading of this manuscript.

\section{References}

1. Marvin, L. F.; Roberts, M. A.; Fay, L. B. Matrix-Assisted Laser Desorption/Ionization Time-of-Flight Mass Spectrometry in Clinical Chemistry. Clin. Chim. Acta 2003, 337(11), 21.

2. Bright, J. J.; Claydon, M. A.; Soufian, M.; Gordon, D. B. Rapid Typing of Bacteria Using Matrix-Assisted Laser Desorption Ionization Time-of-Flight Mass Spectrometry and Pattern Recognition Software. J. Microbiol. Methods 2002, 48, 127-138.

3. Walker, J.; Fox, A. J.; Edwards-Jones, V.; Gordon, D. B. Intact Cell Mass Spectrometry (ICMS) Used to Type MethicillinResistant Staphylococcus aureus: Media Effects and Inter-Laboratory Reproducibility. J. Microbiol. Methods 2002, 48, 117-126.

4. Welham, K. J.; Domin, M. A.; Johnson, K.; Jones, L.; Ashton, D. S. Characterization of Fungal Spores by Laser Desorption/ Ionization Time-of-Flight Mass Spectrometry. Rapid Commun. Mass Spectrom. 2000, 14, 307-310.

5. Yao, Z. P.; Demirev, P. A.; Fenselau, C. Mass SpectrometryBased Proteolytic Mapping for Rapid Virus Identification. Anal. Chem. 2002, 74, 2529-2534.

6. Valentine, N. B.; Wahl, J. H.; Kingsley, M. T.; Wahl, K. L. Direct Surface Analysis of Fungal Species by Matrix-Assisted Laser Desorption/Ionization Mass Spectrometry. Rapid Commun. Mass Spectrom. 2002, 16, 1352-1357.

7. Fenselau, C.; Demirev, P. A. Characterization of Intact Microorganisms by MALDI Mass Spectrometry. Mass Spectrom. Rev. 2001, 20, 157-171.

8. Li, T. Y.; Liu, B. H.; Chen, Y. C. Characterization of Aspergillus Spores by Matrix-Assisted Laser Desorption/Ionization Timeof-Flight Mass Spectrometry. Rapid Commun. Mass Spectrom. 2000, 14, 2393-2400.

9. Domin, M. A.; Welham, K. J.; Ashton, D. S. The Effect of Solvent and Matrix Combinations on the Analysis of Bacteria by Matrix-Assisted Laser Desorption/Ionization Time-ofFlight Mass Spectrometry. Rapid Commun. Mass Spectrom. 1999, 13, 222-226.

10. Dalluge, J. J. Mass Spectrometry for Direct Determination of Proteins in Cells: Applications in Biotechnology and Microbiology. Fresenius J. Anal. Chem. 2000, 366, 701-711.

11. Claydon, M. A.; Davey, S. N.; Edwards-Jones, V.; Gordon, D. B. The Rapid Identification of Intact Microorganisms Using Mass Spectrometry. Nat. Biotechnol. 1996, 14, 1584-1586.

12. Leenders, F.; Stein, T. H.; Kablitz, B.; Franke, P.; Vater, J. Rapid Typing of Bacillus subtilis Strains by Their Secondary Metabolites Using Matrix-Assisted Laser Desorption/Ionization Mass 
Spectrometry of Intact Cells. Rapid Commun. Mass Spectrom. 1999, 13, 943-949.

13. Arnold, R. J.; Karty, J. A.; Ellington, A. D.; Reilly, J. P. Monitoring the Growth of a Bacteria Culture by MALDI-MS of Whole Cells. Anal. Chem. 1999, 71, 1990-1996.

14. Arnold, R. J.; Reilly, J. P. Fingerprint Matching of E. coli Strains with Matrix-Assisted Laser Desorption/Ionization Time-ofFlight Mass Spectrometry of Whole Cells Using a Modified Correlation Approach. Rapid Commun. Mass Spectrom. 1998, 12, 630-636.

15. Wang, Z.; Russon, L.; Li, L.; Roser, D. C.; Long, S. R. Investigation of Spectral Reproducibility in Direct Analysis of Bacteria Proteins by Matrix-Assisted Laser Desorption/Ionization Time-of-Flight Mass Spectrometry. Rapid Commun. Mass Spectrom. 1998, 12, 456-464.

16. Saenz, A. J.; Petersen, C. E.; Valentine, N. B.; Gantt, S. L.; Jarman, K. H.; Kingsley, M. T.; Wahl, K. L. Reproducibility of Matrix-Assisted Laser Desorption/Ionization Time-of-Flight
Mass Spectrometry for Replicate Bacterial Culture Analysis. Rapid Commun. Mass Spectrom. 1999, 13, 1580-1585.

17. Vaidyanathan, S.; Winder, C. L.; Wade, S. C.; Kell, D. B.; Goodacre, R. Sample Preparation in Matrix-Assisted Laser Desorption/Ionization Mass Spectrometry of Whole Bacterial Cells and the Detection of High Mass ( $>20 \mathrm{kDa}$ ) Proteins. Rapid Commun. Mass Spectrom. 2002, 16, 1276-1286.

18. Williams, T. L.; Andrzejewski, D.; Lay, J. O.; Musser, S. M. Experimental Factors Affecting the Quality and Reproducibility of MALDI TOF Mass Spectra Obtained from Whole Bacteria Cells. J. Am. Soc. Mass Spectrom. 2003, 14, 342-351.

19. Schiffrin, E. J.; Blum, S. Food Processing: Probiotic Microorganisms for Beneficial Foods. Curr. Opin. Biotechnol. 2001, 12, 499-502.

20. Krishnamurthy, T.; Ross, P. L. Rapid Identification of Bacteria by Direct Matrix-Assisted Laser Desorption/Ionization Mass Spectrometric Analysis of Whole Cells. Rapid Commun Mass Spectrom. 1996, 10, 1992-1996. 